\title{
PAPER \\ Time-Varying AR Spectral Estimation Using an Indefinite Matrix-Based Sliding Window Fast Linear Prediction
}

Kiyoshi NISHIYAMA $^{\dagger \mathrm{a})}$, Member

\begin{abstract}
SUMMARY A method for efficiently estimating the time-varying spectra of nonstationary autoregressive (AR) signals is derived using an indefinite matrix-based sliding window fast linear prediction (ISWFLP). In the linear prediction, the indefinite matrix plays a very important role in sliding an exponentially weighted finite-length window over the prediction error samples. The resulting ISWFLP algorithm successively estimates the timevarying AR parameters of order $N$ at a computational complexity of $O(N)$ per sample. The performance of the AR parameter estimation is superior to the performances of the conventional techniques, including the YuleWalker, covariance, and Burg methods. Consequently, the ISWFLP-based AR spectral estimation method is able to rapidly track variations in the frequency components with a high resolution and at a low computational cost. The effectiveness of the proposed method is demonstrated by the spectral analysis results of a sinusoidal signal and a speech signal.

key words: spectral estimation, autoregressive model, linear prediction, fast algorithm, sliding window, indefinite matrix, forgetting factor
\end{abstract}

\section{Introduction}

The spectral analysis of signals is widely used in a variety of fields, including engineering and geophysics. Several spectral estimation techniques have been developed based on fitting a measured data set to an assumed model, such as an autoregressive (AR), a moving average (MA), or an autoregressive-moving average (ARMA) model [1][5]. Among the model-based (parametric) methods, the AR model is frequently employed because AR parameter estimation is a well-established problem and is equivalent to solving a system of linear equations. Especially in speech processing, AR modeling is the most popular approach to spectral analysis [5]-[7]. The parameters of an AR model can be estimated in different ways, as seen in the YuleWalker, covariance, Burg, and maximum likelihood estimation methods. These methods are based on the assumption that the signals to be modeled may be approximated as stationary signals over the observation interval. Therefore, they are not inherently highly adaptable to variations in the AR parameters. Additionally, the correlations among the signal samples must be recalculated at each time step during application of successive processing steps. In an effort to address these limitations, several adaptive AR spectral estimation methods have been presented using a recursive least squares (RLS) algorithm with a sliding window [8] or a for-

\footnotetext{
Manuscript received January 17, 2013.

Manuscript revised July 19, 2013.

${ }^{\dagger}$ The author is with the Department of Electrical Engineering and Computer Science, Faculty of Engineering, Iwate University, Morioka-shi, 020-8551 Japan.

a) E-mail: nisiyama@cis.iwate-u.ac.jp

DOI: 10.1587/transfun.E97.A.547
}

getting factor [9]. The sliding window RLS (SWRLS) algorithm is quite suitable for time-varying parameter estimation problems [10]-[12]; however, sliding a finite-length window over a data set imposes a heavy computational burden on the RLS algorithm. The Kalman filter has been applied to adaptive AR modeling of a nonstationary time series [13]. Unfortunately, these methods require arithmetic operations that are proportional to $N^{2}$, namely, $O\left(N^{2}\right)$, per sample to identify an AR model of order $N$. Although fast versions of the RLS and SWRLS algorithms have been developed, these algorithms are quite complicated due to the introduction of two additional error energies [14] or the need for two applications of the fast RLS algorithm per sample [15]. Some researchers have attempted to explicitly model the AR parameters as time-varying [6]; however, the dependency on time must be heuristically defined to approximate the dynamics.

In this paper, we propose a method for successfully estimating the time-varying spectra of nonstationary AR signals using an indefinite matrix-based sliding window fast linear prediction (ISWFLP) algorithm. The ISWFLP algorithm performs the sliding window AR parameter estimation at a computational complexity of $O(N)$ per sample, where the indefinite matrix operation efficiently discards old data during the correlation update. The sliding window used in the SWFLP algorithm is exponentially weighted using a forgetting factor to reinforce the method's adaptability. In general, a high adaptability to variations in the AR parameters can improve both the time and frequency resolutions in the corresponding running spectra. The ISWFLP-based AR spectral estimation method can facilitate the tracking of nonstationary signals with a high frequency resolution. The effectiveness of the proposed method is demonstrated by the spectral analysis results of a frequency-shifted single sinusoid and a speech signal.

The remainder of this paper is organized as follows: Sect. 2 outlines the traditional AR spectral estimation approach. In Sect. 3, the ISWFLP algorithm is derived using the shift properties of the signal correlation matrices, and the algorithm is applied to AR spectral estimation. Section 4 demonstrates the effectiveness of the SWFLP-based AR spectral estimation method using computer simulations. Finally, the conclusions are given in Sect. 5.

\section{Traditional AR Spectral Estimation}

AR spectral estimation procedures consist of two steps. 
Given a data sequence $\left\{u_{k}\right\}$, the parameters of the AR model are estimated, and the power spectral density (PSD) is computed based on these estimates. The AR method is the most frequently used approach among the parametric methods because AR parameter estimation can be easily accomplished by solving a set of linear equations. Generally, the data samples modeled using the AR approach are regarded as the output of a causal, all-pole, discrete-time filter with a white noise input. The current output sample $u_{k}$ may be represented as a weighted sum of $N$ past output samples and a noise term:

$$
u_{k}=-\sum_{n=1}^{N} a(n) u_{k-n}+w_{k}, \quad k=0,1, \cdots, L
$$

where $\{a(n)\}$ are the AR coefficients of the $N$ th order AR process, $w_{k}$ is a zero-mean white noise with a variance equal to $\sigma^{2}$, and the subscript $k$ denotes the index for the sampling time $k T$ with a period $T$.

From the AR parameters, the PSD of the data sequence $\left\{u_{k}\right\}$ may be obtained as

$$
P(f)=\frac{\sigma^{2}}{\left|1+\sum_{n=1}^{N} a(n) e^{j 2 \pi f n}\right|^{2}}
$$

which results in a function of frequency $f$.

A popular approach to estimating the AR parameters is the Yule-Walker method, which is based directly on the following system of linear equations:

$$
\begin{aligned}
& {\left[\begin{array}{cccc}
R_{u}(0) & R_{u}(-1) & \cdots & R_{u}(-N) \\
R_{u}(1) & R_{u}(0) & \cdots & R_{u}(-N+1) \\
\vdots & \vdots & \ddots & \vdots \\
R_{u}(N) & R_{u}(N-1) & \cdots & R_{u}(0)
\end{array}\right]\left[\begin{array}{c}
1 \\
a(1) \\
\vdots \\
a(N)
\end{array}\right]} \\
& =\left[\begin{array}{c}
\sigma^{2} \\
0 \\
\vdots \\
0
\end{array}\right]
\end{aligned}
$$

These equations are characterized by the autocorrelation function $R_{u}(n)=\mathrm{E}\left\{u_{k} u_{k+n}\right\}$ of the data $u_{k}$ where $\mathrm{E}\{\cdot\}$ denotes the expectation. Note that real-valued data are assumed in this work for simplicity. The Levinson-Durbin algorithm efficiently provides a solution to the Yule-Walker equations, with a computational complexity of $O\left(N^{2}\right)$ [1].

Another approach to estimate the AR parameters is the least-squares (LS) AR estimation method, which minimizes the forward prediction error energy:

$$
J=\sum_{k=0}^{L-1}\left|u_{k}-\hat{u}_{k}\right|^{2}, \quad \hat{u}_{k}=-\sum_{n=1}^{N} a(n) u_{k-n}
$$

where $L$ denotes the length of the data. The LS method can be interpreted as providing an approximate solution to the Yule-Walker equations by recognizing that $\frac{1}{L} \sum_{k=0}^{L-n} u_{k} u_{k+n}$ is a finite-sample estimate of $R_{u}(n)$ [2].

\section{The ISWFLP-Based AR Spectral Estimation}

In traditional AR spectral estimation methods, including the Burg method, the coefficients $\{b(n)\}$ of the backward prediction $\hat{u}_{k-N}=-\sum_{n=1}^{N} b(n) u_{k-N+n}$ for a given signal $u_{k}$ are uniquely determined by the forward coefficients $\{a(n)\}$, for example, $b(n)=a(N-n) / a(N)(b(n)=a(n)$ in the Burg method). This implies that the forward linear prediction is completely equivalent to the backward prediction within an observation window. Our key idea in this work is to relax this constraint. To do so, the coefficients required for the forward prediction of the windowed signal are considered to be independent of those required for the backward prediction of the signal. This relaxation will lead to certain improvements in the AR modeling of nonstationary signals. Fortunately, the forward and backward linear predictions provides a closed-form recursive solution to the $A R$ parameter estimation problem.

\subsection{A New Criterion for AR Parameter Estimation}

The above-mentioned objective was accomplished by employing the sum of the forward and backward sliding window linear prediction error powers of the nonstationary signal $u_{k}$ :

$$
\begin{aligned}
J_{k}= & \sum_{i=k-L_{s}+1}^{k} \rho^{k-i}\left|u_{i}+\boldsymbol{U}_{i-1} \boldsymbol{A}_{k}\right|^{2} \\
& +\sum_{i=k-L_{s}+1}^{k} \rho^{k-i}\left|u_{i-N}+\boldsymbol{U}_{i} \boldsymbol{B}_{k}\right|^{2}
\end{aligned}
$$

where the forward and backward linear prediction coefficients may be described in vector form as

$$
\begin{aligned}
\boldsymbol{A}_{k} & =\left[a_{k}(1) a_{k}(2) \cdots a_{k}(N)\right]^{T} \\
\boldsymbol{B}_{k} & =\left[\begin{array}{lll}
b_{k}(N) & \cdots & b_{k}(2) \\
b_{k}(1)
\end{array}\right]^{T}
\end{aligned}
$$

and the corresponding signal samples are given by

$$
\boldsymbol{U}_{i}=\left[u_{i} u_{i-1} \cdots u_{i-N+1}\right] .
$$

This criterion can be expressed using an indefinite matrix as

$$
\begin{aligned}
J_{k}= & \left(\sum_{i=0}^{k} \rho^{k-i}\left|u_{i}+\boldsymbol{U}_{i-1} \boldsymbol{A}_{k}\right|^{2}\right. \\
& \left.-\sum_{i=0}^{k} \rho^{k-i+L_{s}}\left|u_{i-L_{s}}+\boldsymbol{U}_{i-L_{s}-1} \boldsymbol{A}_{k}\right|^{2}\right) \\
& +\left(\sum_{i=0}^{k} \rho^{k-i}\left|u_{i-N}+\boldsymbol{U}_{i} \boldsymbol{B}_{k}\right|^{2}\right. \\
& \left.-\sum_{i=0}^{k} \rho^{k-i+L_{s}}\left|u_{i-L_{s}-N}+\boldsymbol{U}_{i-L_{s}} \boldsymbol{B}_{k}\right|^{2}\right) \\
= & \sum_{i=0}^{k} \rho^{k-i}\left(\left|u_{i}+\boldsymbol{U}_{i-1} \boldsymbol{A}_{k}\right|^{2}\right.
\end{aligned}
$$




$$
\begin{aligned}
& \left.-\rho^{L_{s}}\left|u_{i-L_{s}}+\boldsymbol{U}_{i-L_{s}-1} \boldsymbol{A}_{k}\right|^{2}\right) \\
& +\sum_{i=0}^{k} \rho^{k-i}\left(\left|u_{i-N}+\boldsymbol{U}_{i} \boldsymbol{B}_{k}\right|^{2}\right. \\
& \left.-\rho^{L_{s}}\left|u_{i-L_{s}-N}+\boldsymbol{U}_{i-L_{s}} \boldsymbol{B}_{k}\right|^{2}\right) \\
= & \sum_{i=0}^{k} \rho^{k-i}\left(\boldsymbol{c}_{i}+\boldsymbol{C}_{i-1} \boldsymbol{A}_{k}\right)^{T} \boldsymbol{W}_{s}\left(\boldsymbol{c}_{i}+\boldsymbol{C}_{i-1} \boldsymbol{A}_{k}\right) \\
& +\sum_{i=0}^{k} \rho^{k-i}\left(\boldsymbol{c}_{i-N}+\boldsymbol{C}_{i} \boldsymbol{B}_{k}\right)^{T} \boldsymbol{W}_{s}\left(\boldsymbol{c}_{i-N}+\boldsymbol{C}_{i} \boldsymbol{B}_{k}\right)
\end{aligned}
$$

in which $\boldsymbol{C}_{i}, \boldsymbol{c}_{i}$, and $\boldsymbol{W}_{s}$ are defined, respectively, as

$$
\boldsymbol{C}_{i}=\left[\begin{array}{c}
\boldsymbol{U}_{i} \\
\boldsymbol{U}_{i-L_{s}}
\end{array}\right], \boldsymbol{c}_{i}=\left[\begin{array}{c}
u_{i} \\
u_{i-L_{s}}
\end{array}\right], \boldsymbol{W}_{s}=\left[\begin{array}{cc}
1 & 0 \\
0 & -\rho^{L_{s}}
\end{array}\right]
$$

where $\boldsymbol{C}_{i}$ is a $2 \times N$ signal matrix, $\boldsymbol{c}_{i}$ is the first column vector of $\boldsymbol{C}_{i}, \rho$ is a forgetting factor, $L_{s}$ is the length of the sliding window, $\boldsymbol{W}_{s}$ is an indefinite weight matrix involved in the sliding window processing, and $u_{k}$ is assumed to be a causal signal.

Here, we provide a brief explanation for Eq. (8). For an arbitrary two-dimensional column vector $\left[\begin{array}{l}a \\ b\end{array}\right]$, the difference $a^{2}-b^{2}$ can be represented in a quadratic form of $\left[\begin{array}{cc}1 & 0 \\ 0 & -1\end{array}\right]$, i.e., $a^{2}-b^{2}=\left[\begin{array}{l}a \\ b\end{array}\right]^{T}\left[\begin{array}{cc}1 & 0 \\ 0 & -1\end{array}\right]\left[\begin{array}{l}a \\ b\end{array}\right]$. Applying the indefinite quadratic form to the window sliding step, and taking into account the forgetting effects, one can easily see the transformation from the weighted difference of the squared prediction errors to the quadratic form of $\boldsymbol{W}_{s}$ in (8).

When the coefficients $\boldsymbol{A}_{k}$ and $\boldsymbol{B}_{k}$ are assumed to be mutually independent, the optimal forward prediction coefficients of $\boldsymbol{A}_{k}$, that minimize the criterion $\boldsymbol{J}_{k}$, satisfy the following linear equations:

$$
\boldsymbol{Q}_{k-1} \boldsymbol{A}_{k}=-\boldsymbol{t}_{k}
$$

where

$$
\boldsymbol{Q}_{k-1}=\sum_{i=0}^{k-1} \rho^{k-1-i} \boldsymbol{C}_{i}^{T} \boldsymbol{W}_{s} \boldsymbol{C}_{i}, \boldsymbol{t}_{k}=\sum_{i=0}^{k} \rho^{k-i} \boldsymbol{C}_{i-1}^{T} \boldsymbol{W}_{s} \boldsymbol{c}_{i} .
$$

The forward prediction error power $S_{k}$ may be expressed as

$$
S_{k}=\sum_{i=0}^{k} \rho^{k-i} \boldsymbol{e}_{k, i}^{T} \boldsymbol{W}_{s} \boldsymbol{e}_{k, i}, \quad \boldsymbol{e}_{k, i}=\boldsymbol{c}_{i}+\boldsymbol{C}_{i-1} \boldsymbol{A}_{k} .
$$

For the backward linear prediction, it follows that the optimal $\boldsymbol{B}_{k}$ satisfies

$$
\boldsymbol{Q}_{k} \boldsymbol{B}_{k}=-\underline{\boldsymbol{t}}_{k}
$$

where

$$
\boldsymbol{Q}_{k}=\sum_{i=0}^{k} \rho^{k-i} \boldsymbol{C}_{i}^{T} \boldsymbol{W}_{s} \boldsymbol{C}_{i}, \underline{\boldsymbol{t}}_{k}=\sum_{i=0}^{k} \rho^{k-i} \boldsymbol{C}_{i}^{T} \boldsymbol{W}_{s} \boldsymbol{c}_{i-N} .
$$

The backward prediction error power $\underline{S}_{k}$ may be expressed as

$$
\underline{S}_{k}=\sum_{i=0}^{k} \rho^{k-i} \underline{\boldsymbol{e}}_{k, i}^{T} \boldsymbol{W}_{s} \underline{\boldsymbol{e}}_{k, i}, \quad \underline{\boldsymbol{e}}_{k, i}=\boldsymbol{c}_{i-N}+\boldsymbol{C}_{i} \boldsymbol{B}_{k} .
$$

Now, we briefly discuss the assumption that $\boldsymbol{A}_{k}$ and $\boldsymbol{B}_{k}$ are independent. Recalling (10) and (13), we have

$$
\boldsymbol{A}_{k}=-\boldsymbol{Q}_{k-1}^{-1} \boldsymbol{t}_{k}, \quad \boldsymbol{B}_{k}=-\boldsymbol{Q}_{k}^{-1} \underline{\boldsymbol{t}}_{k}
$$

which reduce to

$$
\boldsymbol{A}=-\boldsymbol{Q}^{-1} \boldsymbol{t}, \quad \boldsymbol{B}=-\boldsymbol{Q}^{-1} \underline{\boldsymbol{t}}
$$

when $u_{k}$ is stationary. From these equations and the results presented in the Appendix, we obtain

$$
\boldsymbol{J} \boldsymbol{B}=-\boldsymbol{J} \boldsymbol{Q}^{-1} \underline{t}=-\boldsymbol{J} \boldsymbol{Q}^{-1} J \boldsymbol{t}=-\boldsymbol{Q}^{-1} \boldsymbol{t}=\boldsymbol{A}
$$

where

$$
\boldsymbol{J}=\left[\begin{array}{cccc}
0 & \cdots & 0 & 1 \\
\vdots & . & 1 & 0 \\
0 & . & . & \vdots \\
1 & 0 & \cdots & 0
\end{array}\right] .
$$

The resulting equation indicates that $b(n)=a(n)$. This relationship between the forward and backward prediction coefficients coincides with that in the Burg method. In nonstationary cases, the forward and backward prediction coefficients $\boldsymbol{A}_{k}$ and $\boldsymbol{B}_{k}$ can not be related rigidly. Therefore, the assumption that $\boldsymbol{A}_{k}$ and $\boldsymbol{B}_{k}$ are independent is most favorable for deriving a new adaptive AR parameter estimation approach. The reasonableness of the assumption will be verified by computer simulations in the following section.

\subsection{A Recursive Solution to the AR Parameter Estimation} Problem

The AR parameter estimation problem may be recursively solved by introducing an auxiliary matrix $\boldsymbol{K}_{k}$, defined by $\boldsymbol{K}_{k}=\boldsymbol{Q}_{k}^{-1} \boldsymbol{C}_{k}^{T}$.

Given the linear prediction coefficients $\boldsymbol{A}_{k}$ and $\boldsymbol{B}_{k}$, the auxiliary matrix $\boldsymbol{K}_{k}$, which satisfies $\boldsymbol{Q}_{k} \boldsymbol{K}_{k}=\boldsymbol{C}_{k}^{T}$, can be recursively updated as follows.

[Proposition 1]

The auxiliary matrix $\boldsymbol{K}_{k}$ can be recursively computed at a computational complexity of $O(N)$ for each time sample as

$$
\left[\begin{array}{c}
\boldsymbol{K}_{k} \\
\mathbf{0}
\end{array}\right]=\left[\begin{array}{c}
\mathbf{0} \\
\boldsymbol{K}_{k-1}
\end{array}\right]+\left[\begin{array}{c}
1 \\
\boldsymbol{A}_{k}
\end{array}\right] \frac{\boldsymbol{e}_{k}^{T}}{S_{k}}-\left[\begin{array}{c}
\boldsymbol{B}_{k} \\
1
\end{array}\right] \underline{\underline{\boldsymbol{e}}_{k}^{T}}
$$

where

$$
\boldsymbol{e}_{k}=\boldsymbol{c}_{k}+\boldsymbol{C}_{k-1} \boldsymbol{A}_{k}, \underline{\boldsymbol{e}}_{k}=\boldsymbol{c}_{k-N}+\boldsymbol{C}_{k} \boldsymbol{B}_{k} .
$$

(Proof)

To take advantage of the shift properties of $\boldsymbol{C}_{k}$ and $\boldsymbol{Q}_{k}$, we introduce the $(N+1) \times 2$ auxiliary matrix $\breve{\boldsymbol{K}}_{k}$ which satisfies 


$$
\breve{\boldsymbol{Q}}_{k} \breve{\boldsymbol{K}}_{k}=\breve{\boldsymbol{C}}_{k}^{T}
$$

where

$$
\begin{aligned}
\breve{\boldsymbol{Q}}_{k} & =\sum_{i=0}^{k} \rho^{k-i} \breve{\boldsymbol{C}}_{i}^{T} \boldsymbol{W}_{s} \breve{\boldsymbol{C}}_{i} \\
\breve{\boldsymbol{C}}_{k} & =\left[\begin{array}{cccc}
u_{k} & u_{k-1} & \cdots & u_{k-N} \\
u_{k-L_{s}} & u_{k-L_{s}-1} & \cdots & u_{k-L_{s}-N}
\end{array}\right] .
\end{aligned}
$$

The matrices $\breve{\boldsymbol{Q}}_{k}$ and $\breve{\boldsymbol{C}}_{k}$ are partitioned as

$$
\begin{aligned}
\breve{\boldsymbol{Q}}_{k} & =\left[\begin{array}{ll}
\boldsymbol{Q}_{k} & \underline{\boldsymbol{t}}_{k} \\
\underline{\boldsymbol{t}}_{k}^{T} & q_{k}^{b}
\end{array}\right]=\left[\begin{array}{cc}
q_{k}^{f} & \boldsymbol{t}_{k} \\
\boldsymbol{t}_{k}^{T} & \boldsymbol{Q}_{k-1}
\end{array}\right] \\
\breve{\boldsymbol{C}}_{k} & =\left[\begin{array}{ll}
\boldsymbol{C}_{k} & \boldsymbol{c}_{k-N}
\end{array}\right]=\left[\begin{array}{ll}
\boldsymbol{c}_{k} & \boldsymbol{C}_{k-1}
\end{array}\right]
\end{aligned}
$$

where

$$
q_{k}^{f}=\sum_{i=0}^{k} \rho^{k-i} \boldsymbol{c}_{i}^{T} \boldsymbol{W}_{s} \boldsymbol{c}_{i}, \quad q_{k}^{b}=\sum_{i=0}^{k} \rho^{k-i} \boldsymbol{c}_{i-N}^{T} \boldsymbol{W}_{s} \boldsymbol{c}_{i-N} .
$$

Then, using the matrix inversion lemma, we can invert $\breve{Q}_{k}$ in two different ways as

$$
\begin{aligned}
\breve{\boldsymbol{Q}}_{k}^{-1} & =\left[\begin{array}{cc}
\boldsymbol{Q}_{k} & \underline{\boldsymbol{t}}_{k}^{T} \\
\underline{\boldsymbol{t}}_{k} & q_{k}^{b}
\end{array}\right]^{-1} \\
& =\left[\begin{array}{cc}
\boldsymbol{Q}_{k}^{-1}+\boldsymbol{Q}_{k}^{-1} \underline{\boldsymbol{t}}_{k} \underline{S}_{k}^{-1} \underline{\boldsymbol{t}}_{k}^{T} \boldsymbol{Q}_{k}^{-1} & -\boldsymbol{Q}_{k}^{-1} \underline{\boldsymbol{t}}_{k} \underline{S}_{k}^{-1} \\
-\underline{S}_{k}^{-1} \underline{\boldsymbol{t}}_{k}^{T} \boldsymbol{Q}_{k}^{-1} & \underline{S}_{k}^{-1}
\end{array}\right] \\
\breve{\boldsymbol{Q}}_{k}^{-1} & =\left[\begin{array}{cc}
q_{k}^{f} & \boldsymbol{t}_{k}^{T} \\
\boldsymbol{t}_{k} & \boldsymbol{Q}_{k-1}
\end{array}\right]^{-1} \\
& =\left[\begin{array}{cc}
S_{k}^{-1} & -S_{k}^{-1} \boldsymbol{t}_{k}^{T} \boldsymbol{Q}_{k-1}^{-1} \\
-\boldsymbol{Q}_{k-1}^{-1} \boldsymbol{t}_{k} S_{k}^{-1} & \boldsymbol{Q}_{k-1}^{-1}+\boldsymbol{Q}_{k-1}^{-1} \boldsymbol{t}_{k} S_{k}^{-1} \boldsymbol{t}_{k}^{T} \boldsymbol{Q}_{k-1}^{-1}
\end{array}\right] .
\end{aligned}
$$

Here it should be noted that $S_{k}$ and $\underline{S}_{k}$ satisfy

$$
S_{k}=q_{k}^{f}-\boldsymbol{t}_{k}^{T} \boldsymbol{Q}_{k-1}^{-1} \boldsymbol{t}_{k}, \underline{S}_{k}=q_{k}^{b}-\underline{\boldsymbol{t}}_{k}^{T} \boldsymbol{Q}_{k}^{-1} \underline{\boldsymbol{t}}_{k} .
$$

Equation (27) allows $\breve{\boldsymbol{K}}_{k}$ to be expressed in terms of $\boldsymbol{B}_{k}$ as

$$
\begin{aligned}
& \breve{\boldsymbol{K}}_{k} \\
& =\left[\begin{array}{cc}
\boldsymbol{Q}_{k}^{-1} & \mathbf{0}^{T} \\
\mathbf{0} & 0
\end{array}\right] \breve{\boldsymbol{C}}_{k}^{T} \\
& +\left[\begin{array}{cc}
\boldsymbol{Q}_{k}^{-1} \underline{\boldsymbol{t}}_{k} \underline{S}_{k}^{-1} \underline{\boldsymbol{t}}_{k}^{T} \boldsymbol{Q}_{k}^{-1} & -\boldsymbol{Q}_{k}^{-1} \underline{\boldsymbol{t}}_{k} \underline{S}_{k}^{-1} \\
-\underline{S}_{k}^{-1} \underline{\boldsymbol{t}}_{k}^{T} \boldsymbol{Q}_{k}^{-1} & \underline{S}_{k}^{-1}
\end{array}\right] \breve{\boldsymbol{C}}_{k}^{T} \\
& =\left[\begin{array}{c}
\boldsymbol{K}_{k} \\
\mathbf{0}
\end{array}\right]+\left[\begin{array}{c}
\boldsymbol{Q}_{k}^{-1} \underline{\boldsymbol{t}}_{k} \underline{S}_{k}^{-1} \underline{\boldsymbol{t}}_{k}^{T} \boldsymbol{K}_{k}-\boldsymbol{Q}_{k}^{-1} \underline{\boldsymbol{t}}_{k} \underline{S}_{k}^{-1} \boldsymbol{c}_{k-N}^{T} \\
-\underline{S}_{k}^{-1} \underline{\boldsymbol{t}}_{k}^{T} \boldsymbol{K}_{k}+\underline{S}_{k}^{-1} \boldsymbol{c}_{k-N}^{T}
\end{array}\right] \\
& =\left[\begin{array}{c}
\boldsymbol{K}_{k} \\
\mathbf{0}
\end{array}\right]+\left[\begin{array}{c}
-\boldsymbol{Q}_{k}^{-1} \underline{\boldsymbol{t}}_{k} \\
1
\end{array}\right] \frac{\boldsymbol{c}_{k-N}^{T}-\underline{\boldsymbol{t}}_{k}^{T} \boldsymbol{K}_{k}}{\underline{S}_{k}} \\
& =\left[\begin{array}{c}
\boldsymbol{K}_{k} \\
\mathbf{0}
\end{array}\right]+\left[\begin{array}{c}
\boldsymbol{B}_{k} \\
1
\end{array}\right] \frac{\boldsymbol{c}_{k-N}^{T}-\underline{\boldsymbol{t}}_{k}^{T} \boldsymbol{Q}_{k}^{-1} \boldsymbol{C}_{k}^{T}}{\underline{S}_{k}} \\
& =\left[\begin{array}{c}
\boldsymbol{K}_{k} \\
\mathbf{0}
\end{array}\right]+\left[\begin{array}{c}
\boldsymbol{B}_{k} \\
1
\end{array}\right] \frac{\left(\boldsymbol{c}_{k-N}+\boldsymbol{C}_{k} \boldsymbol{B}_{k}\right)^{T}}{\underline{S}_{k}},
\end{aligned}
$$

whereas Eq. (28) allows $\breve{\boldsymbol{K}}_{k}$ to be expressed in terms of $\boldsymbol{A}_{k}$ as

$$
\begin{aligned}
\breve{\boldsymbol{K}}_{k} & {\left[\begin{array}{cc}
0 & \mathbf{0}^{T} \\
\mathbf{0} & \boldsymbol{Q}_{k-1}^{-1}
\end{array}\right] \breve{\boldsymbol{C}}_{k}^{T} } \\
& +\left[\begin{array}{cc}
S_{k}^{-1} & -S_{k}^{-1} \boldsymbol{t}_{k}^{T} \boldsymbol{Q}_{k-1}^{-1} \\
-\boldsymbol{Q}_{k-1}^{-1} \boldsymbol{t}_{k} S_{k}^{-1} & \boldsymbol{Q}_{k-1}^{-1} \boldsymbol{t}_{k} S_{k}^{-1} \boldsymbol{t}_{k}^{T} \boldsymbol{Q}_{k-1}^{-1}
\end{array}\right] \breve{\boldsymbol{C}}_{k}^{T} \\
= & {\left[\begin{array}{c}
\mathbf{0} \\
\boldsymbol{K}_{k-1}
\end{array}\right] } \\
& +\left[\begin{array}{c}
-\boldsymbol{Q}_{k-1}^{-1} \boldsymbol{t}_{k} S_{k}^{-1} \boldsymbol{c}_{k}^{T}-S_{k}^{-1} \boldsymbol{\boldsymbol { Q }}_{k-1}^{T} \boldsymbol{K}_{k-1}^{-1} \boldsymbol{t}_{k} S_{k}^{-1} \boldsymbol{t}_{k}^{T} \boldsymbol{K}_{k-1}
\end{array}\right] \\
= & {\left[\begin{array}{c}
\mathbf{0} \\
\boldsymbol{K}_{k-1}
\end{array}\right]+\left[\begin{array}{c}
1 \\
-\boldsymbol{Q}_{k-1}^{-1} \boldsymbol{t}_{k}
\end{array}\right] \frac{\boldsymbol{c}_{k}^{T}-\boldsymbol{t}_{k}^{T} \boldsymbol{K}_{k-1}}{S_{k}} } \\
= & {\left[\begin{array}{c}
\mathbf{0} \\
\boldsymbol{K}_{k-1}
\end{array}\right]+\left[\begin{array}{c}
1 \\
\boldsymbol{A}_{k}
\end{array}\right] \frac{\left(\boldsymbol{c}_{k}+\boldsymbol{C}_{k-1} \boldsymbol{A}_{k}\right)^{T}}{S_{k}} . }
\end{aligned}
$$

Noticing that the right-hand sides of (30) and (31) are equal, we obtain a recursive expression of $\boldsymbol{K}_{k}$ :

$$
\left[\begin{array}{c}
\boldsymbol{K}_{k} \\
\mathbf{0}
\end{array}\right]=\left[\begin{array}{c}
\mathbf{0} \\
\boldsymbol{K}_{k-1}
\end{array}\right]+\left[\begin{array}{c}
1 \\
\boldsymbol{A}_{k}
\end{array}\right] \frac{\boldsymbol{e}_{k}^{T}}{S_{k}}-\left[\begin{array}{c}
\boldsymbol{B}_{k} \\
1
\end{array}\right] \frac{\underline{\boldsymbol{e}}_{k}^{T}}{\underline{S}_{k}}
$$

which is just (20).

[Corollary 1]

The auxiliary matrix $\boldsymbol{K}_{k}$ can be rewritten without $\underline{\boldsymbol{e}}_{k}$ and $\underline{S}_{k}$ as

$$
\boldsymbol{K}_{k}=\boldsymbol{m}_{k}-\boldsymbol{B}_{k} \boldsymbol{\mu}_{k}
$$

where $\boldsymbol{m}_{k}$ and $\boldsymbol{\mu}_{k}$ are defined by

$$
\left[\begin{array}{c}
\boldsymbol{m}_{k} \\
\boldsymbol{\mu}_{k}
\end{array}\right]=\left[\begin{array}{c}
\mathbf{0} \\
\boldsymbol{K}_{k-1}
\end{array}\right]+\left[\begin{array}{c}
1 \\
\boldsymbol{A}_{k}
\end{array}\right] \frac{\boldsymbol{e}_{k}^{T}}{S_{k}} .
$$

(Proof)

Using (33), we can rewrite (20) as

$$
\begin{aligned}
{\left[\begin{array}{c}
\boldsymbol{K}_{k} \\
\mathbf{0}
\end{array}\right] } & =\left[\begin{array}{c}
\boldsymbol{m}_{k} \\
\boldsymbol{\mu}_{k}
\end{array}\right]-\left[\begin{array}{c}
\boldsymbol{B}_{k} \\
1
\end{array}\right] \frac{\underline{\boldsymbol{e}}_{k}^{T}}{\underline{S}_{k}} \\
& =\left[\begin{array}{c}
\boldsymbol{m}_{k}-\frac{\boldsymbol{B}_{k} \underline{\boldsymbol{e}}_{k}^{T}}{\underline{S}_{k}} \\
\boldsymbol{\mu}_{k}-\frac{\underline{\boldsymbol{e}}_{k}^{T}}{\underline{S}_{k}}
\end{array}\right] .
\end{aligned}
$$

Comparing each component of (34) on both sides leads to (32).

Next, we attempt to identify a recursive relation to determine the forward linear prediction coefficients $\boldsymbol{A}_{k}$ and the error power $S_{k}$.

[Proposition 2]

The forward linear prediction coefficients $\boldsymbol{A}_{k}$ and the error power $S_{k}$ can be recursively calculated at a computational complexity of $O(N)$ for each time sample as

$$
\begin{aligned}
& \boldsymbol{A}_{k}=\boldsymbol{A}_{k-1}-\boldsymbol{K}_{k-1} \boldsymbol{W}_{s} \tilde{\boldsymbol{e}}_{k} \\
& S_{k}=\rho S_{k-1}+\boldsymbol{e}_{k}^{T} \boldsymbol{W}_{s} \tilde{\boldsymbol{e}}_{k}
\end{aligned}
$$


where

$$
\tilde{\boldsymbol{e}}_{k}=\boldsymbol{c}_{k}+\boldsymbol{C}_{k-1} \boldsymbol{A}_{k-1}, \quad \boldsymbol{e}_{k}=\boldsymbol{c}_{k}+\boldsymbol{C}_{k-1} \boldsymbol{A}_{k} .
$$

(Proof)

Using the recursive relations of $\boldsymbol{Q}_{k}=\rho \boldsymbol{Q}_{k-1}+\boldsymbol{C}_{k}^{T} \boldsymbol{W}_{s} \boldsymbol{C}_{k}$ and $\boldsymbol{t}_{k}=\rho \boldsymbol{t}_{k-1}+\boldsymbol{C}_{k-1}^{T} \boldsymbol{W}_{s} \boldsymbol{c}_{k}$, we can rewrite the product $\boldsymbol{Q}_{k-1} \boldsymbol{A}_{k-1}$ as

$$
\begin{aligned}
\boldsymbol{Q}_{k-1} \boldsymbol{A}_{k-1} & =\left(\rho \boldsymbol{Q}_{k-2}+\boldsymbol{C}_{k-1}^{T} \boldsymbol{W}_{s} \boldsymbol{C}_{k-1}\right) \boldsymbol{A}_{k-1} \\
& =\rho \boldsymbol{Q}_{k-2} \boldsymbol{A}_{k-1}+\boldsymbol{C}_{k-1}^{T} \boldsymbol{W}_{s} \boldsymbol{C}_{k-1} \boldsymbol{A}_{k-1} \\
& =-\rho \boldsymbol{t}_{k-1}+\boldsymbol{C}_{k-1}^{T} \boldsymbol{W}_{s} \boldsymbol{C}_{k-1} \boldsymbol{A}_{k-1} \\
& =-\boldsymbol{t}_{k}+\boldsymbol{C}_{k-1}^{T} \boldsymbol{W}_{s} \boldsymbol{c}_{k}+\boldsymbol{C}_{k-1}^{T} \boldsymbol{W}_{s} \boldsymbol{C}_{k-1} \boldsymbol{A}_{k-1} \\
& =-\boldsymbol{t}_{k}+\boldsymbol{C}_{k-1}^{T} \boldsymbol{W}_{s}\left(\boldsymbol{c}_{k}+\boldsymbol{C}_{k-1} \boldsymbol{A}_{k-1}\right) .
\end{aligned}
$$

Subtracting the identity

$$
\begin{gathered}
\boldsymbol{Q}_{k-1} \boldsymbol{K}_{k-1} \boldsymbol{W}_{s}\left(\boldsymbol{c}_{k}+\boldsymbol{C}_{k-1} \boldsymbol{A}_{k-1}\right) \\
=\boldsymbol{C}_{k-1}^{T} \boldsymbol{W}_{s}\left(\boldsymbol{c}_{k}+\boldsymbol{C}_{k-1} \boldsymbol{A}_{k-1}\right)
\end{gathered}
$$

from the last equation of (38) on both sides, we obtain

$$
\boldsymbol{Q}_{k-1}\left(\boldsymbol{A}_{k-1}-\boldsymbol{K}_{k-1} \boldsymbol{W}_{s}\left(\boldsymbol{c}_{k}+\boldsymbol{C}_{k-1} \boldsymbol{A}_{k-1}\right)\right)=-\boldsymbol{t}_{k} .
$$

Comparing this equation with (10), we immediately find

$$
\boldsymbol{A}_{k}=\boldsymbol{A}_{k-1}-\boldsymbol{K}_{k-1} \boldsymbol{W}_{s}\left(\boldsymbol{c}_{k}+\boldsymbol{C}_{k-1} \boldsymbol{A}_{k-1}\right) .
$$

On the other hand, from $q_{k}^{f}=\rho q_{k-1}^{f}+\boldsymbol{c}_{k}^{T} \boldsymbol{W}_{s} \boldsymbol{c}_{k}$, we can find a recursive expression for $S_{k}$ as

$$
\begin{aligned}
S_{k}= & q_{k}^{f}+\boldsymbol{t}_{k}^{T} \boldsymbol{A}_{k} \\
= & \rho q_{k-1}^{f}+\boldsymbol{c}_{k}^{T} \boldsymbol{W}_{s} \boldsymbol{c}_{k}+\left(\rho \boldsymbol{t}_{k-1}+\boldsymbol{C}_{k-1}^{T} \boldsymbol{W}_{s} \boldsymbol{c}_{k}\right)^{T} \\
& \times\left[\boldsymbol{A}_{k-1}-\boldsymbol{K}_{k-1} \boldsymbol{W}_{s}\left(\boldsymbol{c}_{k}+\boldsymbol{C}_{k-1} \boldsymbol{A}_{k-1}\right)\right] \\
= & \rho\left(q_{k-1}^{f}+\boldsymbol{t}_{k-1}^{T} \boldsymbol{A}_{k-1}\right)+\boldsymbol{c}_{k}^{T} \boldsymbol{W}_{s}\left(\boldsymbol{c}_{k}+\boldsymbol{C}_{k-1} \boldsymbol{A}_{k-1}\right) \\
& -\boldsymbol{t}_{k}^{T} \boldsymbol{K}_{k-1} \boldsymbol{W}_{s}\left(\boldsymbol{c}_{k}+\boldsymbol{C}_{k-1} \boldsymbol{A}_{k-1}\right) \\
= & \rho S_{k-1}+\boldsymbol{c}_{k}^{T} \boldsymbol{W}_{s}\left(\boldsymbol{c}_{k}+\boldsymbol{C}_{k-1} \boldsymbol{A}_{k-1}\right) \\
& -\boldsymbol{t}_{k}^{T} \boldsymbol{Q}_{k-1}^{-1} \boldsymbol{C}_{k-1}^{T} \boldsymbol{W}_{s}\left(\boldsymbol{c}_{k}+\boldsymbol{C}_{k-1} \boldsymbol{A}_{k-1}\right) \\
= & \rho S_{k-1}+\boldsymbol{c}_{k}^{T} \boldsymbol{W}_{s}\left(\boldsymbol{c}_{k}+\boldsymbol{C}_{k-1} \boldsymbol{A}_{k-1}\right) \\
& +\boldsymbol{A}_{k}^{T} \boldsymbol{C}_{k-1}^{T} \boldsymbol{W}_{s}\left(\boldsymbol{c}_{k}+\boldsymbol{C}_{k-1} \boldsymbol{A}_{k-1}\right) \\
= & \rho S_{k-1}+\left(\boldsymbol{c}_{k}+\boldsymbol{C}_{k-1} \boldsymbol{A}_{k}\right)^{T} \boldsymbol{W}_{s}\left(\boldsymbol{c}_{k}+\boldsymbol{C}_{k-1} \boldsymbol{A}_{k-1}\right) .
\end{aligned}
$$

Note that $\tilde{e}_{k}$ and $e_{k}$ are called the pseudoforward and forward prediction errors, respectively.

Finally, we attempt to identify a recursive relation to determine the backward linear prediction coefficients $\boldsymbol{B}_{k}$.

[Proposition 3]

The backward linear prediction coefficients $\boldsymbol{B}_{k}$ can be recursively calculated at a computational complexity of $O(N)$ for each time sample as

$$
\boldsymbol{B}_{k}=\frac{\boldsymbol{B}_{k-1}-\boldsymbol{m}_{k} \boldsymbol{W}_{s} \underline{\tilde{\boldsymbol{e}}}_{k}}{1-\boldsymbol{\mu}_{k} \boldsymbol{W}_{s} \underline{\tilde{\boldsymbol{e}}}_{k}}
$$

where

$$
\begin{aligned}
& \tilde{\boldsymbol{e}}_{k}=\boldsymbol{c}_{k-N}+\boldsymbol{C}_{k} \boldsymbol{B}_{k-1} \\
& {\left[\begin{array}{c}
\boldsymbol{m}_{k} \\
\boldsymbol{\mu}_{k}
\end{array}\right]=\left[\begin{array}{c}
\mathbf{0} \\
\boldsymbol{K}_{k-1}
\end{array}\right]+\left[\begin{array}{c}
1 \\
\boldsymbol{A}_{k}
\end{array}\right] \frac{\boldsymbol{e}_{k}^{T}}{S_{k}} .}
\end{aligned}
$$

(Proof)

The equation $\boldsymbol{Q}_{k-1} \boldsymbol{B}_{k-1}=-\underline{\boldsymbol{t}}_{k-1}$ can be rewritten according to the recursive relations for $\boldsymbol{Q}_{k}$ and $\underline{\boldsymbol{t}}_{k}$ as follows:

$$
\begin{aligned}
& \boldsymbol{Q}_{k-1} \boldsymbol{B}_{k-1}=-\underline{\boldsymbol{t}}_{k-1} \\
& \left(\rho^{-1} \boldsymbol{Q}_{k}-\rho^{-1} \boldsymbol{C}_{k}^{T} \boldsymbol{W}_{s} \boldsymbol{C}_{k}\right) \boldsymbol{B}_{k-1} \\
& \quad=-\left(\rho^{-1} \underline{\boldsymbol{t}}_{k}-\rho^{-1} \boldsymbol{C}_{k}^{T} \boldsymbol{W}_{s} \boldsymbol{c}_{k-N}\right) \\
& \rho^{-1} \boldsymbol{Q}_{k} \boldsymbol{B}_{k-1}-\rho^{-1} \boldsymbol{C}_{k}^{T} \boldsymbol{W}_{s}\left(\boldsymbol{C}_{k} \boldsymbol{B}_{k-1}+\boldsymbol{c}_{k-N}\right) \\
& \quad=-\rho^{-1} \underline{\boldsymbol{t}}_{k} \\
& \boldsymbol{Q}_{k} \boldsymbol{B}_{k-1}-\boldsymbol{C}_{k}^{T} \boldsymbol{W}_{s} \tilde{\boldsymbol{e}}_{k}=-\underline{\boldsymbol{t}}_{k} \\
& \boldsymbol{Q}_{k}\left(\boldsymbol{B}_{k-1}-\boldsymbol{Q}_{k}^{-1} \boldsymbol{C}_{k}^{T} \boldsymbol{W}_{s} \underline{\tilde{e}}_{k}\right)=-\underline{\boldsymbol{t}}_{k} \\
& \boldsymbol{Q}_{k}\left(\boldsymbol{B}_{k-1}-\boldsymbol{K}_{k} \boldsymbol{W}_{s} \underline{\tilde{\boldsymbol{e}}}_{k}\right)=-\underline{\boldsymbol{t}}_{k} .
\end{aligned}
$$

Comparing the last equation with (13), we have

$$
\boldsymbol{B}_{k}=\boldsymbol{B}_{k-1}-\boldsymbol{K}_{k} \boldsymbol{W}_{s} \underline{\tilde{e}}_{k} .
$$

Substituting $\boldsymbol{K}_{k}=\boldsymbol{m}_{k}-\boldsymbol{B}_{k} \boldsymbol{\mu}_{k}$ into (47) provides

$$
\boldsymbol{B}_{k}=\boldsymbol{B}_{k-1}-\left(\boldsymbol{m}_{k}-\boldsymbol{B}_{k} \boldsymbol{\mu}_{k}\right) \boldsymbol{W}_{s} \underline{\tilde{e}}_{k} .
$$

Rearranging this equation in terms of $\boldsymbol{B}_{k}$, we obtain a feasible recursive expression for $\boldsymbol{B}_{k}$ :

$$
\boldsymbol{B}_{k}=\frac{\boldsymbol{B}_{k-1}-\boldsymbol{m}_{k} \boldsymbol{W}_{s} \underline{\tilde{\boldsymbol{e}}}_{k}}{1-\boldsymbol{\mu}_{k} \boldsymbol{W}_{s} \underline{\tilde{e}}_{k}}
$$

Note that $\underline{\tilde{\boldsymbol{e}}}_{k}$, defined by (44), is referred to as the pseudobackward prediction error.

In summary, a closed form of a recursive algorithm (the ISWFLP algorithm) for efficiently estimating the AR parameters is described using Propositions 1-3 and Corollary 1 :

$$
\begin{aligned}
& \boldsymbol{A}_{k}=\boldsymbol{A}_{k-1}-\boldsymbol{K}_{k-1} \boldsymbol{W}_{s} \tilde{\boldsymbol{e}}_{k} \\
& S_{k}=\rho S_{k-1}+\boldsymbol{e}_{k}^{T} \boldsymbol{W}_{s} \tilde{\boldsymbol{e}}_{k}
\end{aligned}
$$

where

$$
\begin{aligned}
& \boldsymbol{K}_{k}=\boldsymbol{m}_{k}-\boldsymbol{B}_{k} \boldsymbol{\mu}_{k} \\
& \boldsymbol{B}_{k}=\left[\boldsymbol{B}_{k-1}-\boldsymbol{m}_{k} \boldsymbol{W}_{s} \tilde{\boldsymbol{e}}_{k}\right]\left[1-\boldsymbol{\mu}_{k} \boldsymbol{W}_{s} \tilde{\boldsymbol{e}}_{-}\right]^{-1} \\
& {\left[\begin{array}{c}
\boldsymbol{m}_{k} \\
\boldsymbol{\mu}_{k}
\end{array}\right]=\left[\begin{array}{c}
\mathbf{0} \\
\boldsymbol{K}_{k-1}
\end{array}\right]+\left[\begin{array}{c}
1 \\
\boldsymbol{A}_{k}
\end{array}\right] \frac{\boldsymbol{e}_{k}^{T}}{S_{k}}} \\
& \tilde{\underline{\boldsymbol{e}}}_{k}=\boldsymbol{c}_{k-N}+\boldsymbol{C}_{k} \boldsymbol{B}_{k-1} \\
& \boldsymbol{e}_{k}=\boldsymbol{c}_{k}+\boldsymbol{C}_{k-1} \boldsymbol{A}_{k}, \tilde{\boldsymbol{e}}_{k}=\boldsymbol{c}_{k}+\boldsymbol{C}_{k-1} \boldsymbol{A}_{k-1}
\end{aligned}
$$

in which the recursive relations are initialized by $\boldsymbol{K}_{-1}=$ $\mathbf{0}, \boldsymbol{A}_{-1}=\mathbf{0}, S_{-1}=1 / \varepsilon_{0}$, and $\boldsymbol{B}_{-1}=\mathbf{0}$, respectively. The forgetting factor is set to $0<\rho \leq 1$, the indefinite matrix $\boldsymbol{W}_{s}$ is a $2 \times 2$ diagonal matrix with diagonal entries 1 and $-\rho^{L_{s}}$, and the length of the sliding window is chosen to be $L_{s}>N$ 
Table 1 Computational complexity of the ISWFLP algorithm; the division of the $N$-dimensional vector by a scalar is performed using one division and $N$ multiplications.

\begin{tabular}{|c|ccc|}
\hline Computation & Multiplications & Additions & Divisions \\
\hline \hline$\tilde{\boldsymbol{e}}_{k}=\boldsymbol{c}_{k}+\boldsymbol{C}_{k-1} \boldsymbol{A}_{k-1}$ & $2 N$ & $2 N$ & 0 \\
\hline $\boldsymbol{A}_{k}=\boldsymbol{A}_{k-1}-\boldsymbol{K}_{k-1} \boldsymbol{W}_{s} \tilde{\boldsymbol{e}}_{k}$ & $2 N+2$ & $2 N$ & 0 \\
\hline $\boldsymbol{e}_{k}=\boldsymbol{c}_{k}+\boldsymbol{C}_{k-1} \boldsymbol{A}_{k}$ & $2 N$ & $2 N$ & 0 \\
\hline$S_{k}=\rho S_{k-1}+\boldsymbol{e}_{k}^{T} \boldsymbol{W}_{s} \tilde{\boldsymbol{e}}_{k}$ & 5 & 2 & 0 \\
\hline $\boldsymbol{0}$ \\
{$\left[\begin{array}{c}\boldsymbol{m}_{k} \\
\boldsymbol{\mu}_{k}\end{array}\right]\left[\begin{array}{c}\mathbf{0} \\
\boldsymbol{K}_{k-1}\end{array}\right]+\left[\begin{array}{c}1 \\
\boldsymbol{A}_{k}\end{array}\right] \underline{\boldsymbol{e}}_{k}^{T}$} \\
$S_{k}$ & $2 N+2$ & $2 N$ & 1 \\
\hline$\underline{\boldsymbol{e}}_{k}=\boldsymbol{c}_{k-N}+\boldsymbol{C}_{k} \boldsymbol{B}_{k-1}$ & $2 N$ & $2 N$ & 0 \\
\hline $\boldsymbol{B}_{k}=\left[\boldsymbol{B}_{k-1}-\boldsymbol{m}_{k} \boldsymbol{W}_{s} \tilde{\boldsymbol{e}}_{k}\right]\left[1-\boldsymbol{\mu}_{k} \boldsymbol{W}_{s} \tilde{\boldsymbol{e}}_{k}\right]^{-1}$ & $3 N+6$ & $2 N+2$ & 1 \\
\hline \hline $\boldsymbol{K}_{k}=\boldsymbol{m}_{k}-\boldsymbol{B}_{k} \boldsymbol{\mu}_{k}$ & $2 N$ & $2 N$ & 0 \\
\hline Total number & $15 N+15$ & $14 N+4$ & 2 \\
\hline
\end{tabular}

where $N$ is the order of the linear predictor.

The corresponding algorithm equations and computational complexity are summarized in Table 1 .

\subsection{AR Spectral Estimation}

Substituting the AR coefficients $\left\{a_{k}(n)\right\}$ of $\boldsymbol{A}_{k}$ and the error power $S_{k}$ estimated by the ISWFLP algorithm into (2), we can immediately obtain the PSD of the nonstationary signal at time $k$ as

$$
P_{k}(f)=\frac{S_{k}}{\left|1+\sum_{n=1}^{N} a_{k}(n) e^{j 2 \pi f n}\right|^{2}} .
$$

Evolving $P_{k}(f)$ over time $k$ provides a time-frequency representation (running spectra or spectrogram) of the signal. This procedure is referred to as the ISWFLP-based AR spectral estimation method. The ISWFLP-based method will provide a high-resolution time-frequency analysis of the signal.

\section{Simulations}

The performance of the ISWFLP algorithm was evaluated for its ability to estimate the coefficients of a nonstationary AR process. After the fundamental evaluation, the ISWFLP algorithm was applied to an AR spectral estimation problem of a sinusoidal signal and a speech signal. All calculations were performed using MATLAB. The signal sampling frequency was set to $8 \mathrm{kHz}$ throughout the simulations, and the sliding window size was chosen to be $L_{s}=256$ based on a stationary range in speech processing.

\subsection{AR Parameter Estimation}

The performance of the ISWFLP algorithm was investigated by considering a signal example in which the AR coefficients of the vowel /e/ suddenly changed to those of the vowel $/ \mathrm{a} /$ at time $k=2000$, and then returned to the former coefficients at time $k=3000$. The AR coefficients $\left\{a_{k}(n)\right\}$

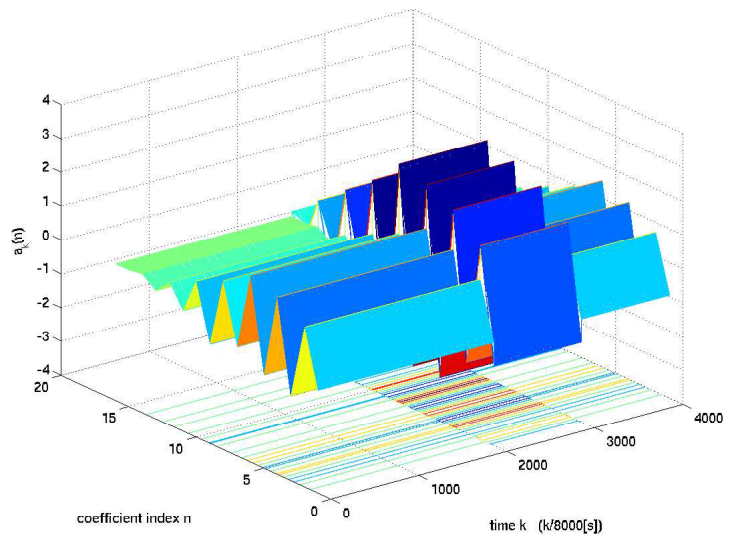

Fig. 1 AR coefficients of order 16 to be tracked.

varied with time $k$, as shown in Fig. 1, where an AR process of order 16 was used to model the vowels.

Figure 2 compares the Levinson-Durbin algorithm and the SWFLP algorithm in their capacities for tracking the changes in the AR coefficients. The Levinson-Durbin algorithm was applied to an original data set (the reproduced AR signal corresponding to the vowels) and the 256-point Hamming-windowed data. The first component $a_{k}(1)$ of the AR coefficients is plotted in Fig. 3 to facilitate a closer examination. Figure 4 plots the squared errors $\sum_{n=1}^{16}\left(a_{k}(n)-\right.$ $\left.a_{k}^{*}(n)\right)^{2}$ as a function of time $k$ where $a_{k}^{*}(n)$ denotes the true values of $a_{k}(n)$. These results demonstrated that the ISWFLP algorithm tracked the changes in the AR coefficients more accurately than the Levinson-Durbin algorithm. The Hamming window smoothed the tracking trajectories of the AR coefficients obtained from the Levinson-Durbin algorithm; however, the analysis based on windows yielded an output that overshot the actual values of trajectories.

\subsection{AR Spectral Estimation}

The performance of the proposed ISWFLP-based method was evaluated for AR spectral estimation of a single sinu- 


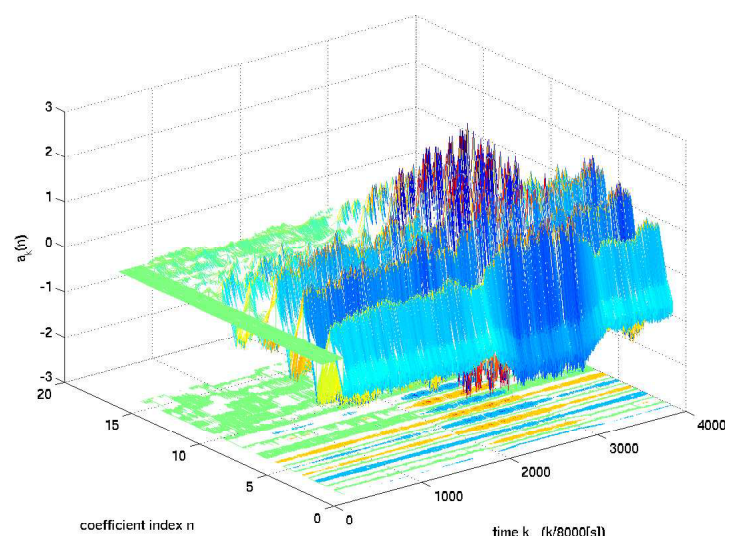

(a)

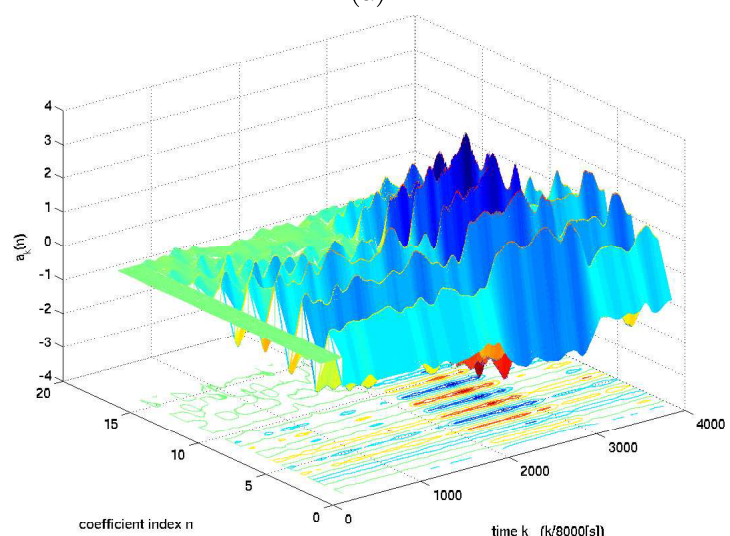

(b)

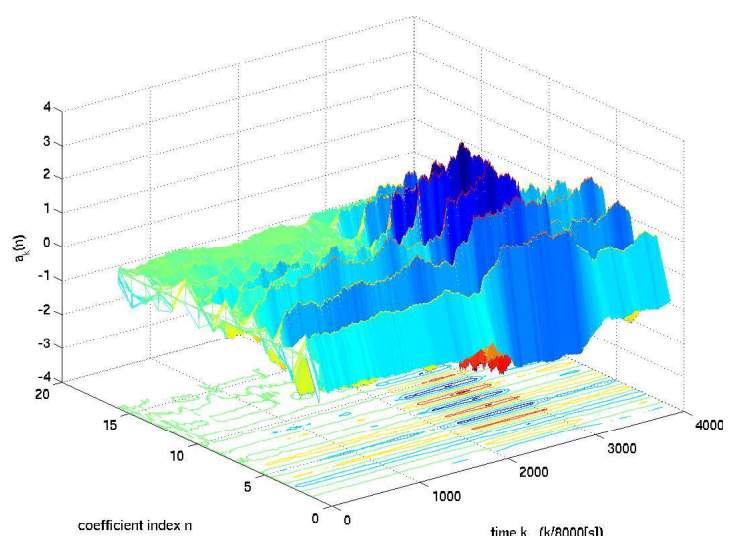

(c)

Fig. 2 Trajectories of the AR coefficients estimated using the LevinsonDurbin algorithm or the ISWFLP algorithm; (a) the results obtained by applying the Levinson-Durbin algorithm to the original data, (b) the results obtained by applying the Levinson-Durbin algorithm to the Hammingwindowed data, (c) the results obtained by applying the ISWFLP algorithm with $\rho=0.999$ and $\varepsilon_{0}=100.0$, where $N=16, L_{s}=256$.

soidal signal and a speech signal.

First, we consider a noisy single sinusoidal signal with a frequency shift:

$$
\begin{gathered}
u_{k}=\sin \left(2 \pi f_{k} t_{k}\right)+v_{k}, f_{k}=\left\{\begin{array}{r}
625[\mathrm{~Hz}], k \leq 2000 \\
2500[\mathrm{~Hz}], k>2000
\end{array}\right. \\
t_{k}=k T, k=0,1,2, \cdots, 4000
\end{gathered}
$$

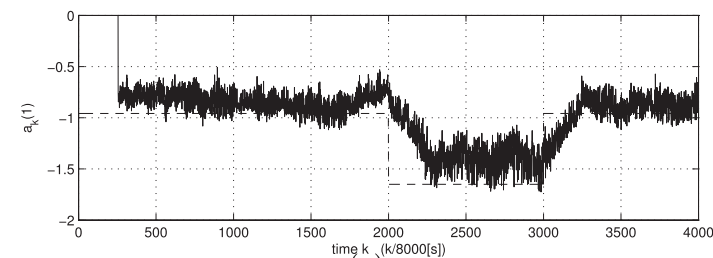

(a)
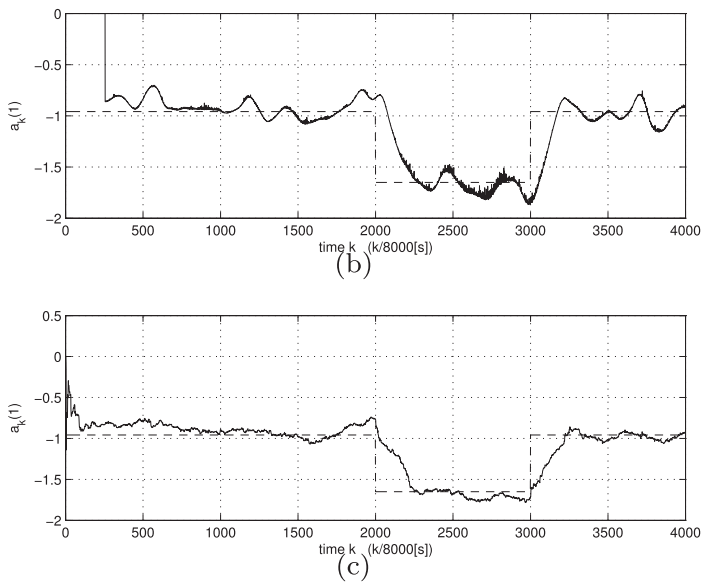

Fig. 3 Estimates of the first AR coefficient $a_{k}(1)$ obtained using the Levinson-Durbin algorithm or the ISWFLP algorithm; (a) the results obtained by applying the Levinson-Durbin algorithm to the original data, (b) the results obtained by applying the Levinson-Durbin algorithm to the Hamming-windowed data, (c) the results obtained by applying the ISWFLP algorithm with $\rho=0.999$ and $\varepsilon_{0}=100.0$, where $N=16, L_{s}=256$.
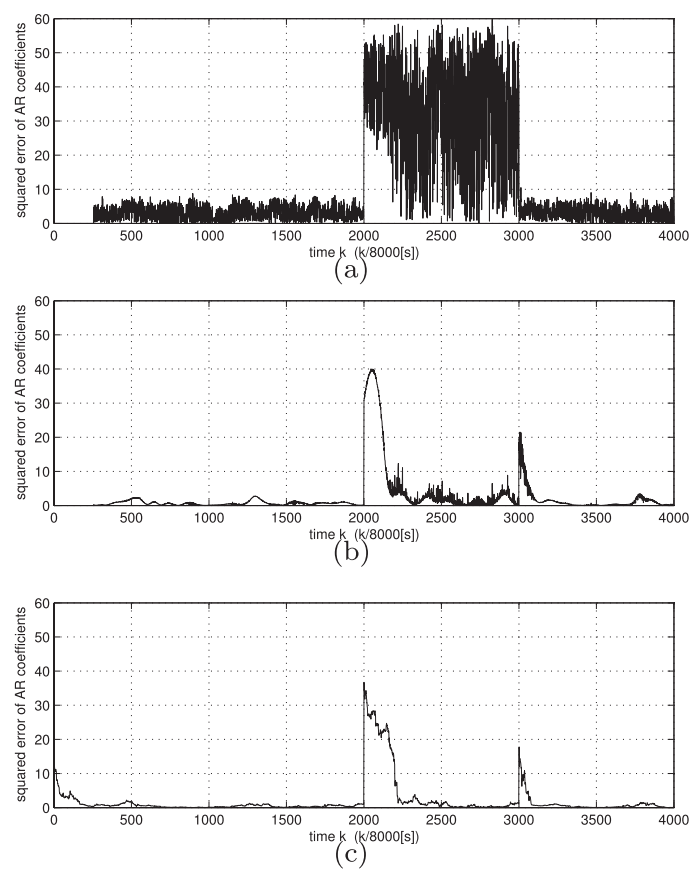

Fig. 4 Squared errors of the AR coefficients obtained using the Levinson-Durbin algorithm or the ISWFLP algorithm; (a) the results obtained by applying the Levinson-Durbin algorithm to the original data, (b) the results obtained by applying the Levinson-Durbin algorithm to the Hamming-windowed data, (c) the results obtained by applying the ISWFLP algorithm with $\rho=0.999$ and $\varepsilon_{0}=100.0$, where $N=16, L_{s}=256$. 


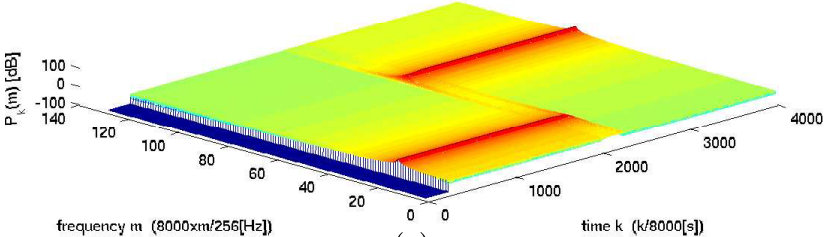

(a)

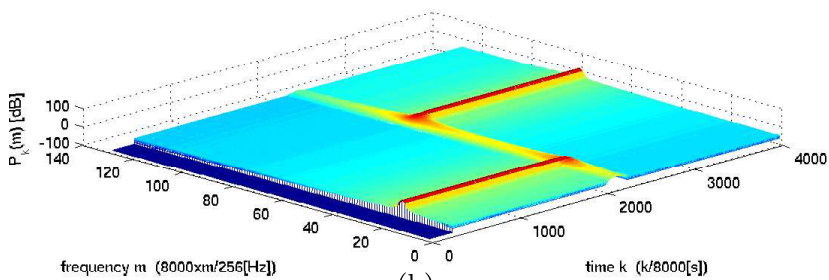

(b)

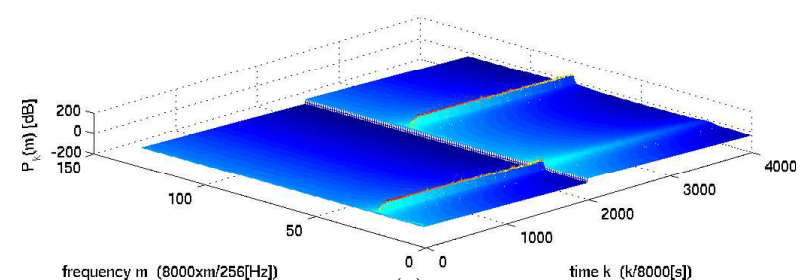

(c)

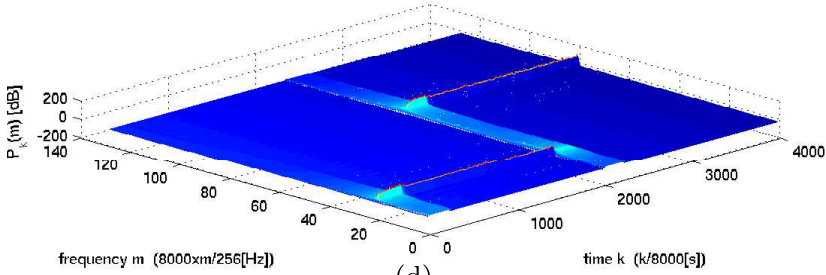

(d)

Fig.5 Running spectra of the single sinusoidal signal for each method; (a) the results obtained by applying the Yule-Walker method to the original data, (b) the results obtained by applying the Yule-Walker method to the Hamming-windowed data, (c) the results obtained by applying the Kalman filter-based method, (d) the results obtained by applying the ISWFLP-based method with $\rho=0.999$ and $\varepsilon_{0}=10.0$, where $N=64, L_{s}=256$.

where $v_{k}$ is a stationary white Gaussian noise with zero mean and a standard deviation of $\sigma_{v}=0.04$ and the sampling pe$\operatorname{riod}$ is $T=1 / 8000 \mathrm{~s}$. Sinusoid analysis is very important because the nonstationary nature of a speech signal may be caused by variations in the signal source. Note that a small amount of observation noise was used to test the noise sensitivity of each method.

Figure 5 compares the performances of the YuleWalker method using the Levinson-Durbin algorithm, the Kalman filter-based method, and the proposed ISWFLPbased method for running spectra estimation of the single sinusoidal signal. The spectra obtained using the ISWFLPbased method and the Kalman filter-based method were very sharp with a small line width, and one major spectral peak was always observed, even during the transient period. By contrast, the spectra obtained using the Yule-Walker method included spurious frequency components with broad line widths during the transient period. This result was also sup-

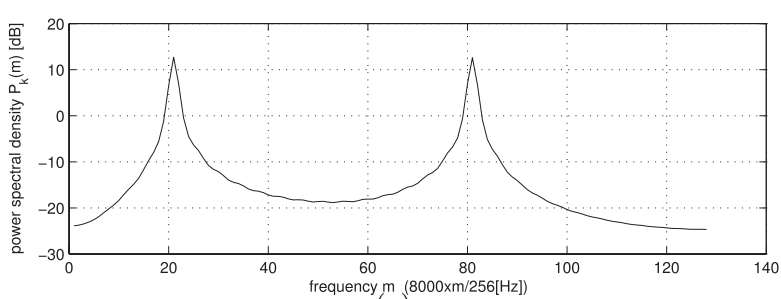

(a)

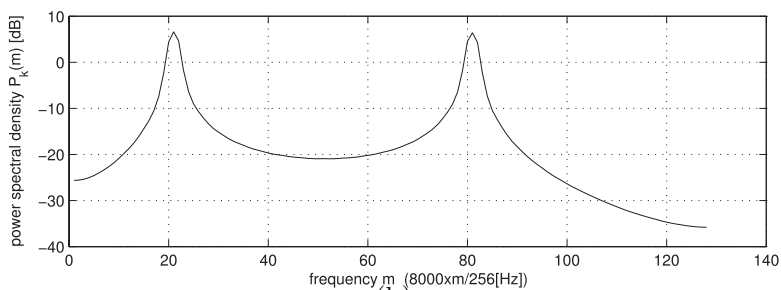

(b)

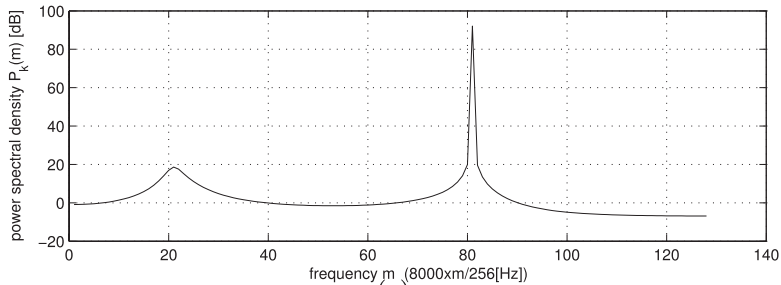

(c)

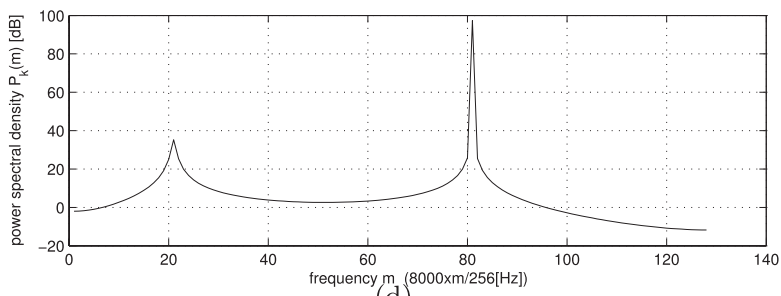

(d)

Fig. 6 Power spectral densities of the single sinusoidal signal at time $k=$ 2128 for each method; (a) the results obtained by applying the Yule-Walker method to the original data, (b) the results obtained by applying the YuleWalker method to the Hamming-windowed data, (c) the results obtained by applying the Kalman filter-based method, (d) the results obtained by applying the ISWFLP-based method with $\rho=0.999$ and $\varepsilon_{0}=10.0$, where $N=64, L_{s}=256$.

ported by the power spectral densities of the sinusoidal signal at time $k=2128$, as shown in Fig. 6. The Yule-Walker power spectral densities included two peaks with comparable magnitudes where one spectral peak should have been present.

The adaptability of the Kalman filter-based method was improved by introducing a zero-mean system noise with covariance $\sigma_{w}^{2} \boldsymbol{I}_{N}$ into the state-space model where $\sigma_{w}^{2}$ was set to $10^{-4}$ and $\boldsymbol{I}_{N}$ is the $N$-dimensional identity matrix. In the ISWFLP-based method, the values of $N, L_{s}$, and $\rho$ were empirically determined to provide the desired time and frequency resolutions in the running spectra. Then the frequency resolution improved as $N$ increased, and the time resolution improved as $L_{s}$ and $\rho$ decreased. It should be noted here that decreasing $L_{s}$ and $\rho$ risks nonsingularity of the matrix $\boldsymbol{Q}_{k}$. Therefore, the forgetting factor $\rho$ must be carefully selected within a range of 0.995 to 0.999 .

Figures 7 and 8 show the AR coefficient estimates and 


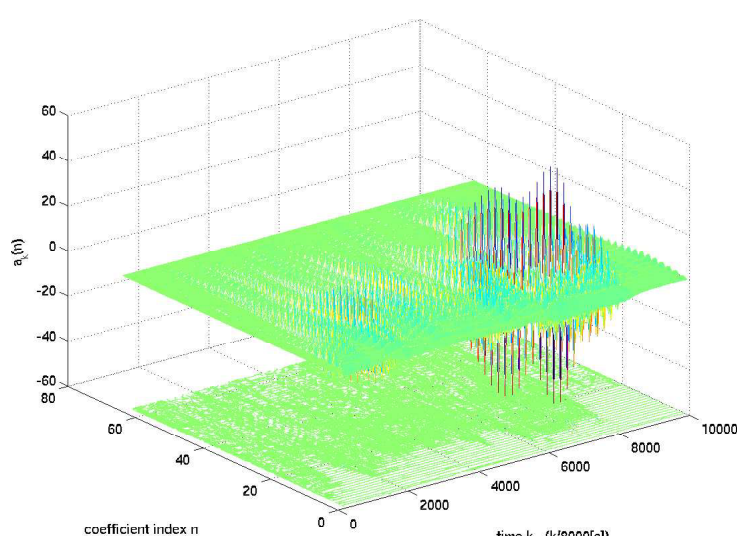

(a)

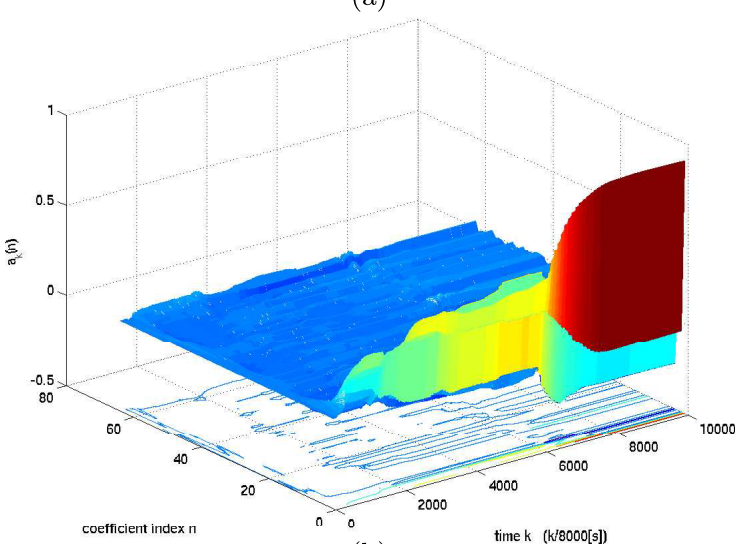

(b)

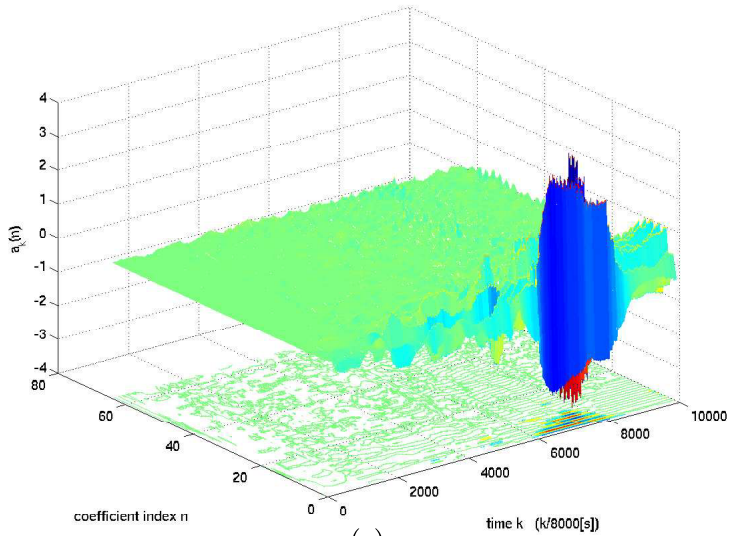

(c)

Fig. 7 Estimated AR coefficients of the speech signal for each method; (a) the results obtained by applying the Yule-Walker method to the Hamming-windowed data, (b) the results obtained by applying the Kalman filter-based method, (c) the results obtained by applying the ISWFLP-based method with $\rho=0.999$ and $\varepsilon_{0}=10.0$, where $N=64, L_{s}=256$.

the corresponding spectrograms obtained by analyzing a speech signal corresponding to the spoken phrase "this is easy for us" " using the Yule-Walker method, the Kalman filter-based method, and the ISWFLP-based method. Here, the maximum value of each spectrogram was normalized to one to improve readability, and the spectral magnitudes were expressed in decibels.

In this example, the ISWFLP-based method provided a spectrogram with clear harmonic structures that may be sim-

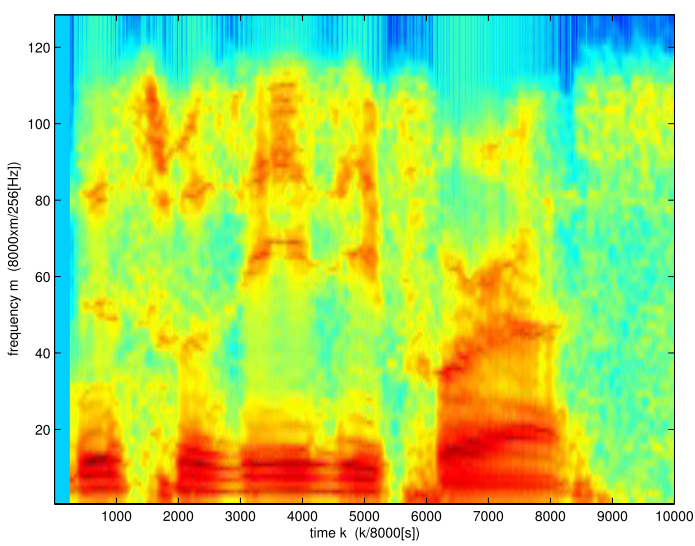

(a)
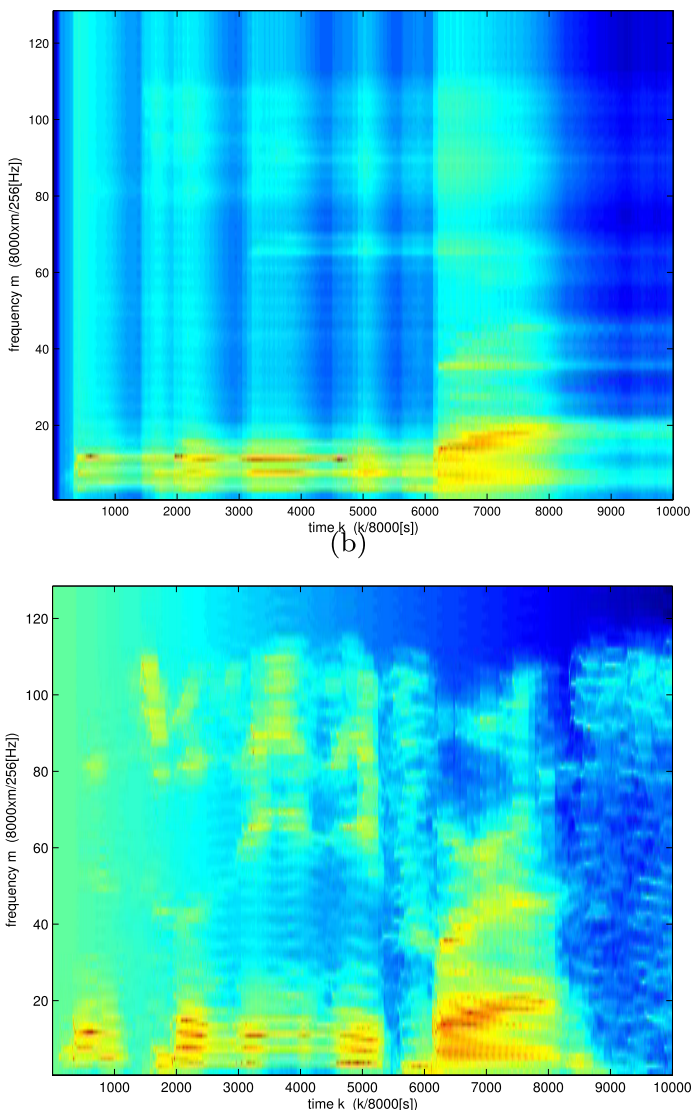

(c)

Fig. 8 Spectrograms of the speech signal for each method; (a) the results obtained by applying the Yule-Walker method to the Hamming-windowed data, (b) the results obtained by applying the Kalman filter-based method, (c) the results obtained by applying the ISWFLP-based method with $\rho=$ 0.999 and $\varepsilon_{0}=10.0$, where $N=64, L_{s}=256$.

ilar to a peripheral auditory spectrogram [16]. The formant trajectories were found to be sharply defined. The AR coefficients estimated using the ISWFLP-based method differed significantly from those obtained using the Yule-Walker method, which provides values within a limited range. In the Yule-Walker method, variations in the frequency com-

Note that the signal data is available on the Web at http://research.nii.ac.jp/src/en/UME-ERJ.html. 
ponents broadened the spectral line width. Additionally, the sliding window processing introduced time lags into the spectra, even when a Hamming window was used. Unfortunately, the sinusoid analysis in the previous example suggests that the Yule-Walker spectrogram may contain numerous spurious frequency components.

\section{Conclusion}

We have proposed an ISWFLP-based method for successfully estimating the time-varying spectra of nonstationary AR signals. In the derivation of the proposed method, the indefinite matrix operation played an important role in the sliding window processing of the adaptive AR parameter estimation. The ISWFLP-based AR spectral estimation method enabled nonstationary signals to be tracked rapidly with a high frequency resolution at a low computational cost. Computer simulations demonstrated that the ISWFLPbased method efficiently estimated the time-varying spectra of a spoken English sentence with a high resolution in both the time and frequency domains, providing an auditory spectrogram that sharply defined the formant trajectories.

The applicability of the ISWFLP-based method will be investigated in the context of a variety of practical problems, including the analysis of earthquake signals.

\section{References}

[1] S.M. Kay and S.L. Marple, "Spectrum analysis - A modern perspective," Proc. IEEE, vol.69, no.11, pp.1380-1419, 1981

[2] Stoica and Moses, Spectral Analysis of Signals, Pearson Prentice Hall, 2005.

[3] S. Haykin, Adaptive Filter Theory, 3rd ed., Prentice-Hall, 1996.

[4] E.D. Ubeyli and I. Guler, "Selection of optimal AR spectral estimation method for internal carotid arterial Doppler signals using cramer-rao Bound," Computers \& Electrical Engineering, vol.30, no.7, pp.491-508, 2004.

[5] J.R. Deller, J.H.L. Hansen, and J.G. Proakis, Discrete-Time Processing of Speech Signals, 2nd ed., Wiley-IEEE Press, 1999.

[6] M.G. Hall, A.V. Oppenheim, and A.S. Willsky, "Time-varying parametric modeling of speech," Signal Process., vol.5, no.3, pp.267285, 1983.

[7] Y. Ephraim and D. Malah, "Speech enhancement using a minimum mean-square error short-time spectral amplitude estimator," IEEE Trans. Acoust. Speech Signal Process., vol.32, no.6, pp.1109-1121, 1984

[8] N. Martin, "An AR spectral analysis of non-stationary signals," Signal Process., vol.10, no.1, pp.61-74, 1986

[9] Y.S. Cho, S.B. Kim, and E.J. Powers, "Time-varying spectral estimation using AR models with variable forgetting factors," IEEE Trans. Signal Process., vol.39, no.6, pp.1422-1426, 1991.

[10] B.-Y. Choi and Z. Bien, "Sliding windowed weighted recursive least-squares method for parameter estimation," Electron. Lett., vol.25, no.20, pp.1381-1382, 1989.

[11] H. Liu and Z. He, "A sliding-exponential window RLS adaptive filtering algorithm: Properties and applications," Signal Process. vol.45, pp.357-368, 1995.

[12] K. Nishiyama, "A new formalism of the sliding window recursive least squares algorithm and its fast version," IEICE Trans. Fundamentals, vol.E94-A, no.6, pp.1394-1400, June 2011

[13] M. Arnold, X.H.R. Milner, H. Witte, R. Bauer, and C. Braun, "Adaptive AR modeling of nonstationary time series by means of Kalman filtering,” IEEE Trans. Biomed. Eng., vol.45, no.5, pp.553-562, 1998.

[14] L. Marple, "A new autoregressive spectrum analysis algorithm," IEEE Trans. Acoust. Speech Signal Process., vol.28, no.4, pp.441454, 1980.

[15] J.M. Cioffi and T. Kailath, "Windowed fast transversal filters adaptive algorithms with normalization," IEEE Trans. Acoust. Speech Signal Process., vol.33, no.3, pp.607-625, 1985.

[16] R.M. Stern and N. Morgan, "Hearing is believing," IEEE Signal Process. Mag., vol.29, no.6, pp.34-43, 2012.

\section{Appendix: Properties of the Toeplitz Matrix}

The following proposition and corollary are useful for analyzing the AR parameter estimation.

[Proposition A.1]

The Toeplitz matrix $\boldsymbol{Q}$ is invariant under the exchange of a row and a column in the reverse order, i.e.,

$J Q J=Q$.

(Proof)

Each element of the Toeplitz matrix is expressed with $Q_{i j}=Q_{|i-j|}$. Thus, each element of $\tilde{\boldsymbol{Q}}=\boldsymbol{J} \boldsymbol{Q}^{-1} \boldsymbol{J}$ is equal to that of $\boldsymbol{Q}$ because $\tilde{Q}_{i j}=Q_{(N-j+1)(N-i+1)}=$ $Q_{|(N-j+1)-(N-i+1)|}=Q_{|i-j|}=Q_{i j}$. This leads to $\boldsymbol{J} \boldsymbol{Q J}=\boldsymbol{Q}$.

[Corollary A.1]

The inverse of the Toeplitz matrix $\boldsymbol{Q}^{-1}$ is invariant under the exchange of a row and a column in the reverse order, i.e.,

$J Q^{-1} J=Q^{-1}$.

(Proof)

Inverting both sides of $\boldsymbol{J} \boldsymbol{Q J}=\boldsymbol{Q}$, respectively, we obtain

$$
J Q^{-1} J=Q^{-1}
$$

because $\boldsymbol{J}^{-1}=\boldsymbol{J}$.

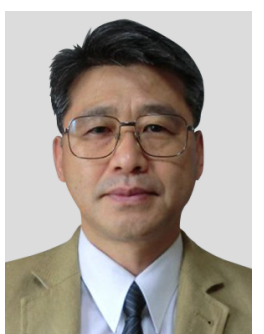

Kiyoshi Nishiyama was born in Tokyo, Japan in 1957. He received the M.E. degree in electrical engineering from Chiba University in 1985 and the degree of Dr. Eng. from Tokyo Institute of Technology in 1991. He joined the Department of Computer and Information Sciences, Iwate University in 1998. He is currently a Professor. His research interests are in estimation, optimization, and machine learning. 